\title{
Effects of the upper cutoff of the electron distribution on the light curves of GRB afterglows
}

\author{
Petropoulou Maria ${ }^{1}$, Mastichiadis Apostolos ${ }^{2}$ and Piran Tsvi ${ }^{3}$ \\ 1,2 Section of Astronomy, Astrophysics and Mechanics, Department of Physics, University of Athens \\ Athens, Greece \\ ${ }^{3}$ The Racah Institute of Physics, The Hebrew University \\ Jerusalem, Israel \\ E-mail: maroulaakiegmail.com amastichephys.uoa.gr, \\ tsviephys.huji.ac.il
}

\begin{abstract}
We investigate the behaviour of the frequency centered light curves expected within the standard model of Gamma Ray Bursts after taking into account the maximum electron energy $\left(\gamma_{\max }\right)$ as an additional free parameter.

First we consider cases where $\gamma_{\max }$ is constant throughout the evolution of the afterglow and present the different X-ray lightcurve morphologies obtained for various ratios of the maximum to the miminum electron Lorenzt factor.

As a second step we generalize our results by assuming that the maximum electron Lorentz factor varies with radius as a result of variable acceleration and loss timescales. We will discuss some of the results giving emphasis on the shape of the expected X-ray lightcurves and hardness ratios.
\end{abstract}

25th Texas Symposium on Relativistic Astrophysics - TEXAS 2010

December 06-10, 2010

Heidelberg, Germany 


\section{The Model}

We assume the standard GRB afterglow model (see e.g. [ए2] for a review) where electrons are being accelerated and radiate their energy in the Relativistic Blast Wave (RBW) formed by the initial explosion. By solving numerically the kinetic equations of electrons and photons which form a system of two coupled partial differential equations we can calculate self consistently the time evolution of the electron distribution and photon spectra taking into account both synchrotron and SSC losses [Q, [3]]. In cases where electrons are injected with a power-law distribution between two cutoffs, $\gamma_{\min }$ and $\gamma_{\max }$, with the upper cutoff being not much greater than the lower one, the obtained $\mathrm{X}$-ray light curves show in general (i) an early-time fast decaying power law segment which is then followed by (ii) a 'plateau'-like and then by (iii) a late-time decaying power law segment. Phase (i) corresponds to the time interval during which the $\mathrm{X}$-ray window is dominated by the exponential cutoff of the synchrotron spectrum. The plateau phase (ii) begins as the synchrotron component decays and the lower energy part of the SSC spectrum, i.e the one below the peak in $v F_{v}$ units, enters the observing window. Finally, phase (iii) of the light curve can be attributed to the normal decay of the SSC component. The above are exemplified in Fig. 四, which shows snapshots of multiwavelength spectra for a GRB lying at $z=1$. Specifically, the first snapshot corresponds to phase (i), snapshots labeled with $t=10^{3} \mathrm{~s}$ and $t=10^{4} \mathrm{~s}$ correspond to phase (ii) and the two remaining ones to phase (iii) described above. Other parameters used for this plot are the total isotropic energy $E_{0}=10^{54} \mathrm{ergs}$, the initial Lorentz factor of the RBW $\Gamma_{0}=400$, the external particle density $n_{0}=1$ part $/ \mathrm{cm}^{3}$, the slope of the injected electron spectrum $p=2.3$ and the upper cutoff of the electron distribution $\gamma_{\max }=2.24 \times 10^{4}$. For the dimensionless parameters $\varepsilon_{\mathrm{B}}$ and $\varepsilon_{\mathrm{e}}$ we used values of 0.001 and 0.025 respectively. The corresponding $X$-ray lightcurve is shown in panel (a) of Fig. \.

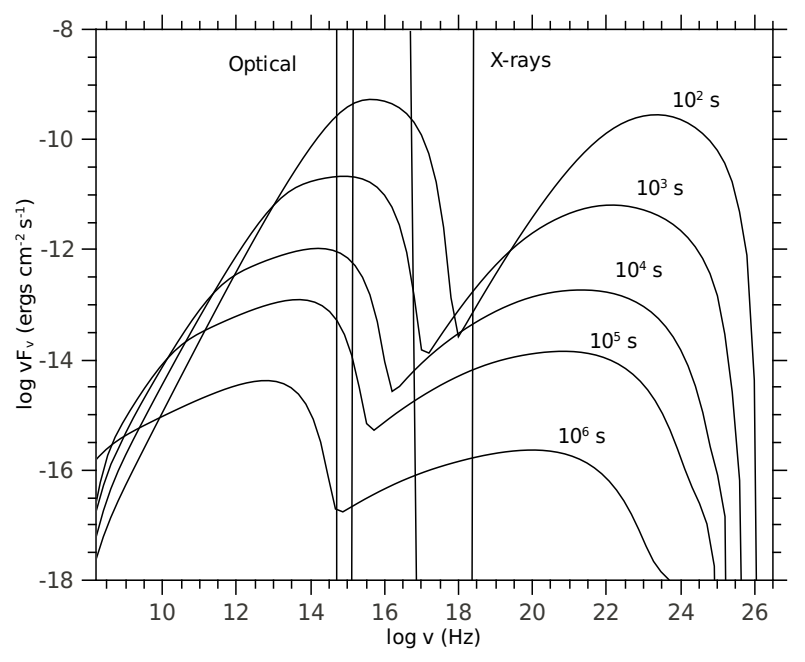

Figure 1: Multiwavelength spectra at observer times from $10^{2} \mathrm{~s}$ to $10^{6} \mathrm{~s}$ (top to bottom). For the parameters used see text. Vertical lines define the X-ray and optical windows that correspond to the observing energy range of XRT (0.3-10) keV and UVOT (170-650) nm respectively.

It is important to emphasize that the close relation we find between X-ray light curves showing 
a plateau phase and a choise of $\gamma_{\max }$ not much greater than $\gamma_{\min }$ is the main thing that differentiates our model [14]] from other works [ [甘], [8, [1]].
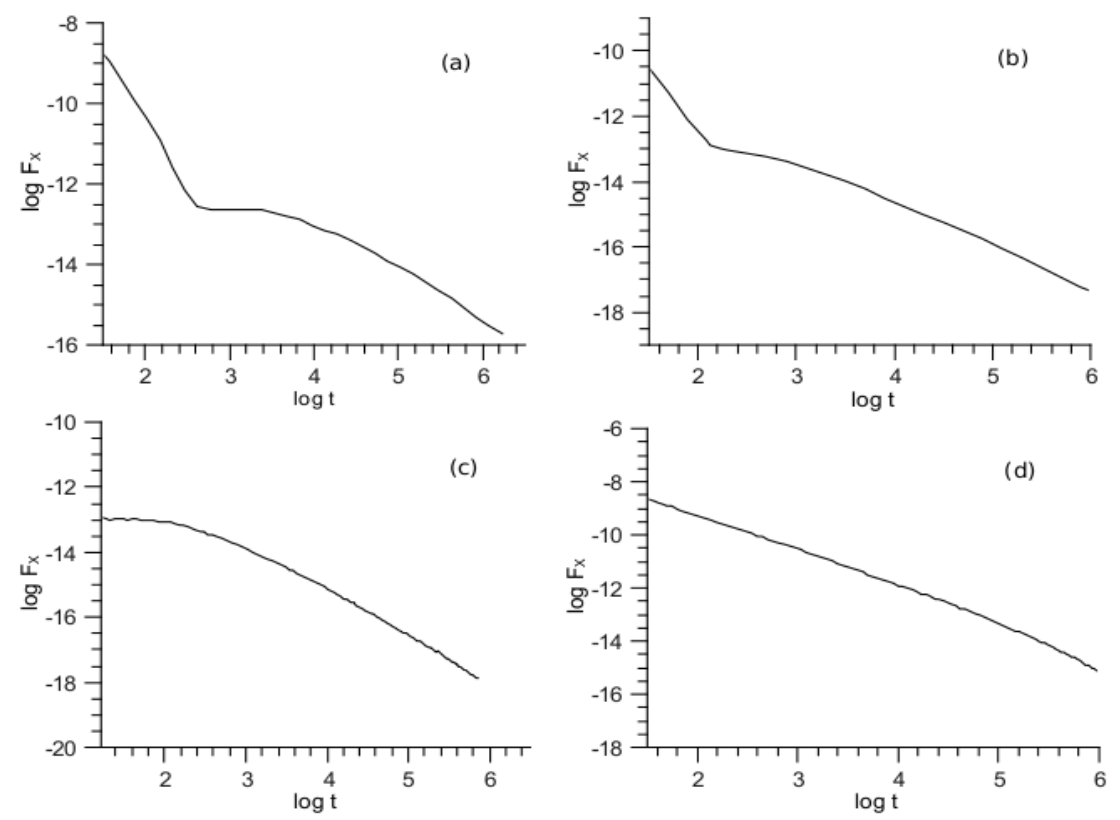

Figure 2: Different light curve morphologies obtained using the numerical code described in [ए]3] for different values of $\gamma_{\max }$ and $\varepsilon_{\mathrm{e}}$. Light curves from each panel can be tentatively compared to the corresponding ones of Fig. 9 from [䧃]. The vector of parameters used is: $\left(E_{0}, \Gamma_{0}, n_{0}, \varepsilon_{\mathrm{B}}, p\right)=\left(10^{54}, 400,1,10^{-3}, 2.3\right)$ where all quantities are expressed in cgs units. The values of pairs $\left(\varepsilon_{\mathrm{e}}, \gamma_{\max }\right)$ used in panels (a) to (d) are respectively: $\left(2.5 \times 10^{-2}, 2.24 \times 10^{4}\right),\left(5 \times 10^{-3}, 1.4 \times 10^{4}\right),\left(3.2 \times 10^{-3}, 6.3 \times 10^{3}\right)$ and $\left(10^{-2}, 10^{6}\right)$.

\section{Effects of $\gamma_{\max }$ on X-ray light curves}

\subsection{Constant $\gamma_{\max }$}

First we consider $\gamma_{\max }$ to be constant throughout the evolution of the afterglow. For fixed values of the standard afterglow model parameters $E_{0}, n_{0}, \Gamma_{0}, \varepsilon_{\mathrm{B}}$ and $p$ we can produce different $\mathrm{X}$-ray light curve morphologies (see Fig. [l) by just choosing different values of $\left(\gamma_{\max }, \varepsilon_{\mathrm{e}}\right)$. Variation of $\varepsilon_{\mathrm{e}}$ implies that the ratio of $\gamma_{\max }$ to $\gamma_{\min }$ changes. For example, the ratio $\gamma_{\max } / \gamma_{\min , 0}$ equals to 1.5 , 8.5 and 500 for the cases presented respectively in panels (a), (b) and (d) of Fig. 1. Here, index ' 0 ' denotes the initial value of the minimum electron Lorentz factor.

Motivated by the similarities of the qualitative behaviour between our model light curves and those observed, we used our model in order to fit, as an indicative example, the X-ray light curve and hardness ratio of GRB 060512 lying at a redshift $z=0.4428$ [四]. The parameters used for the fit shown in Fig. [3] are: $E_{0}=6.5 \times 10^{53} \mathrm{ergs}, \Gamma_{0}=220, n_{0}=100 \mathrm{part} / \mathrm{cm}^{3}, p=2.3, \varepsilon_{\mathrm{e}}=2.7 \times 10^{-2}$, $\varepsilon_{\mathrm{B}}=10^{-5}$ and $\gamma_{\max }=6.5 \times 10^{4}$. Some of the XRT data (for $190 \lesssim t \lesssim 330 \mathrm{~s}$ ) were excluded while fitting the light curve, as they can probably be attributed to an X-ray flare. 

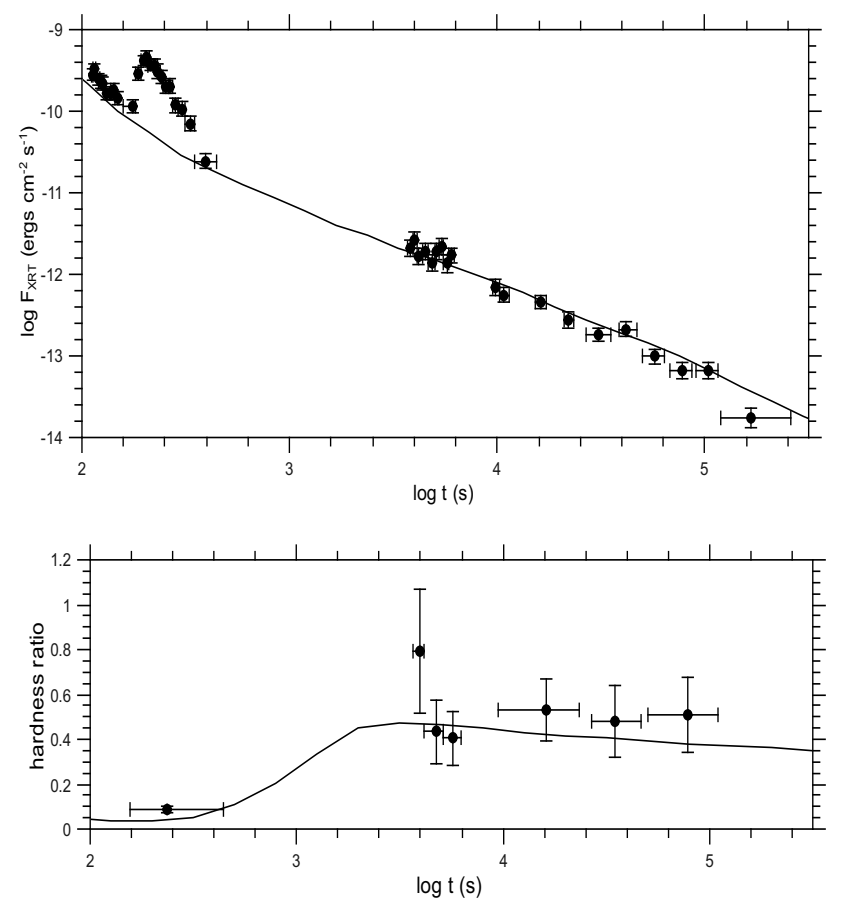

Figure 3: Top panel shows the XRT light curve (points) of GRB 060512 [ [1], 囵] and our model-fit light curve (black solid line), while the panel below shows the evolution of the hardness ratio. For the fitting parameters used see text.

\subsection{Variable $\gamma_{\max }$}

Next we extend our calculations by allowing the injected electron distribution function to be of the form $\gamma^{-p} \mathrm{e}^{-4 \gamma / \gamma_{\max }}$ for $\gamma>\gamma_{\min }$, i.e. we assume a more gradual upper turnover than in the previous case. This can be a more realistic approach in cases where some acceleration mechanism accelerates electrons to high energies and the maximum cutoff is obtained by balancing the power gained and lost due to the acceleration and the synchrotron emission of the particles respectively [10, [1, [5]]. This allows $\gamma_{\max }$ to be a function of radius since the assumption of a constant acceleration timescale yields $\gamma_{\max } \propto B^{-1 / 2}$ and the magnetic field strength varies inherently with distance. When the usual relation $B(r) \propto \varepsilon_{\mathrm{B}}^{1 / 2} \Gamma(r)$ is used, one finds readily that $\gamma_{\max }=$ $\gamma_{\max , 0}\left(r / r_{\mathrm{d}}\right)^{3 / 4}$, where $r_{\mathrm{d}}$ is the deceleration radius of the RBW and $\gamma_{\max , 0}$ is the assumed constant value of $\gamma_{\max }$ for $r<r_{\mathrm{d}}$. The observed maximum synchrotron frequency $v_{\mathrm{s}, \max } \propto \Gamma B \gamma_{\max }^{2}$, in this case decreases as $\propto r^{-3 / 2}$ instead of $\propto r^{-3}$ in the case of a constant $\gamma_{\max }$. It is interesting also to examine a case of a faster increasing maximum Lorentz factor. This has been done more for comparison reasons rather than being based on physical grounds. Thus, we assumed an upper cutoff of the form $\gamma_{\max }=\gamma_{\max , 0}\left(r / r_{\mathrm{d}}\right)^{9 / 8}$ which leads to an even slower decrease of $v_{\mathrm{s}, \max } \propto r^{-3 / 4}$.

Even under these assumptions we can find initial parameters which will produce X-ray light curves with a plateau phase. Some of their qualitative characteristics however change. This is demonstrated in Fig. 团, where the X-ray light curves and hardness ratios (each normalized to its maximum value) of the three aforementioned cases are plotted. In our model the early-time declining part of the X-ray light curve is attributed to the synchrotron emission of the external shock 

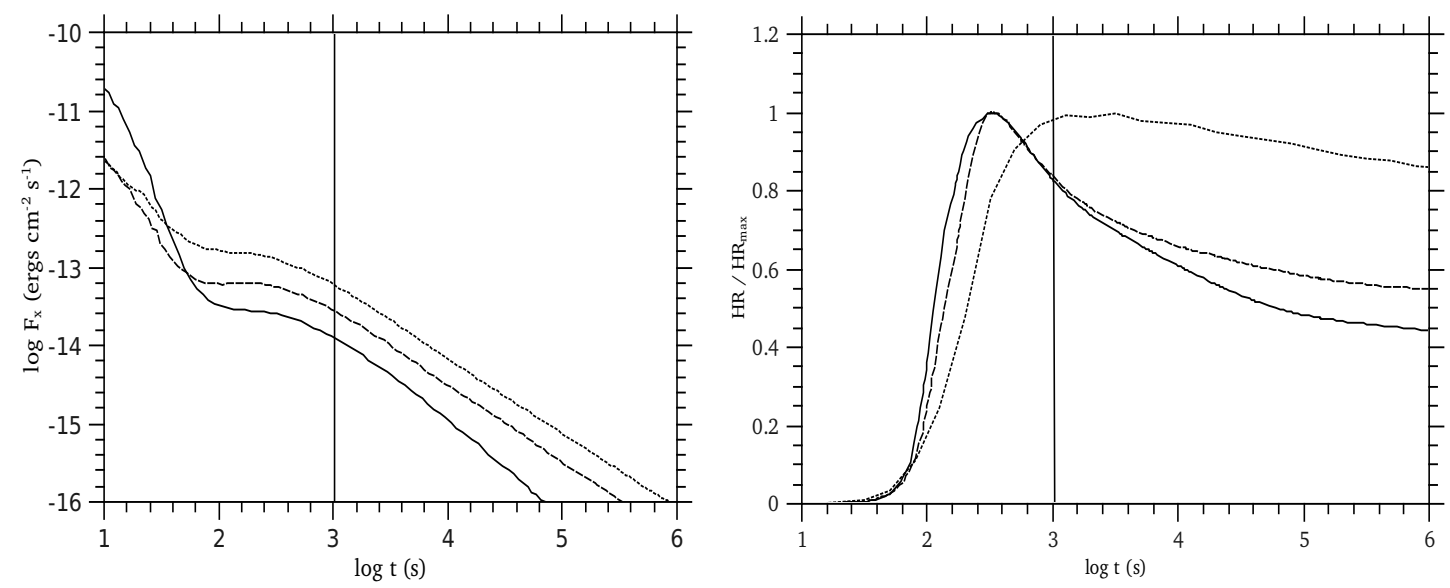

Figure 4: X-ray light curves (left panel) and corresponding hardness ratios (right panel) obtained using constant (solid lines) and a radius dependent $\gamma_{\max }$ as $\sim r^{3 / 4}$ (dashed lines) and $\sim r^{9 / 8}$ (dotted lines). The injected electron distribution is also different: power law without (solid lines) and with (dashed and dotted lines) exponential cutoff. In the left panel the dotted lightcurve is plotted with an offset of +0.35 in logarithmic units of flux for clarity reasons. Parameters used are: $E_{0}=10^{54} \mathrm{ergs}, \Gamma_{0}=400, n_{0}=1 \mathrm{part} / \mathrm{cm}^{-3}, \varepsilon_{\mathrm{B}}=10^{-5}$, $\varepsilon_{\mathrm{e}}=10^{-2}, p=2.3$ and $\gamma_{\max , 0}=10^{4}$. At $t=100 \mathrm{~s}$ the maximum Lorentz factors are approximately the same for all three cases.

and corresponds to the exponential cutoff of the synchrotron spectrum. The shape of the cutoff, resulting from the convolution of the synchrotron emissivity with the electron distibution function, becomes more gradual and broader in the case where an exponential upper cutoff is added to the injected electron distribution. In such a case the early-time flux decay becomes shallower, i.e. $F_{x} \sim t^{-1.37}$ and $\sim t^{-1.35}$ (dashed and dotted lines respectively), which is less steep than most of the observational data showing early X-ray afterglow flux decay of the form $F_{x} \sim t^{-\alpha}$ with $2 \lesssim \alpha \lesssim 5$ [III, 团]. Note however that this result depends critically on the assumed injected electron distribution function. On the other hand the late-time behaviour of the hardness ratio, i.e after the end of the plateau phase, becomes more consistent with the observations, which show no significant late-time spectral evolution [ए], ㅁ] ]. This is demonstrated at the right panel of Fig. 田. For times $t>10^{3} \mathrm{~s}$ the change of the hardness ratio with time becomes less prominant as the dependance of $\gamma_{\max }$ on radius increases, i.e. from a constant $\gamma_{\max }$ (solid line) to one varying as $r^{9 / 8}$ (dotted line).

We have shown that the maximum Lorentz factor of the electrons as well as the functional form of the distribution near the cutoff affect significantly the shape of the obtained X-ray lightcurves and hardness ratios. In our present work however, we have assumed a priori both the functional form of $\gamma_{\max }$ with respect to the radius and the shape of the electron distribution near the upper cutoff. A self-consistent treatment of the problem will be considered in a future work, by adding a suitable acceleration term to the kinetic equation of the electrons.

\section{References}

[1] J. S. Bloom, R. J. Foley, D. Kocevski, \& D. Perley, $G C N$, 5217, 1B 
[2] N. R. Butler \& D. Kocevski, 2007, ApJ, 668, 400 [astro-ph/0702638]

[3] O. C. de Jager, A. K. Harding, P. F. Michelson et al. 1996, ApJ, 457, 253

[4] D. Eichler \& J. Granot, 2006, ApJ, 641, L5 [astro-ph/0509857]

[5] P. A. Evans, A. P. Beardmore, K. L. Page, et al. 2007, A\&A, 469, 379 [astro-ph/0704.0128]

[6] P. A. Evans, A. P. Beardmore, K. L. Page, et al. 2009 MNRAS, 397, 1177 [astro-ph/0812.3662]

[7] Y. Fan, T. Piran, R. Narayan \& D. Wei, 2008, MNRAS, 384, 1483 [astro-ph/0704.2063]

[8] G. Ghisellini, G. Ghirlanda, L. Nava \& C. Firmani, 2007 ApJ, 658, L75 [astro-ph/0701430]

[9] E. Liang, B. Zhang \& B. Zhang, 2007, ApJ, 670, 565 [astro-ph/0705.1373]

[10] A. F. Heavens \& K. Meisenheimer, 1987, MNRAS, 225, 335

[11] J. A. Nousek, C. Kouveliotou, D. Grupe, et al. 2006, ApJ, 642, 389 [astro-ph/0508332]

[12] T. Piran, 2004, RvMP, 76, 1143 [astro-ph/0405503]

[13] M. Petropoulou \& A. Mastichiadis, 2009, A\&A, 507, 509 [astro-ph/0909.0208]

[14] M. Petropoulou, A. Mastichiadis \& T. Piran, $A \& A$ submitted

[15] L. Stawarz \& V. Petrosian, 2008, ApJ, 681, 1725 [arxiv: 0803.0989]

[16] S. Vaughan, M. R. Goad, A. P. Beardmore, et al. 2006, ApJ, 638, 920 [astro-ph/0510677]

[17] B. Zhang, Y. Z. Fan, J. Dyks, et al. 2006, ApJ, 642, 354 [astro-ph/0508321] 